\title{
Audit of the workload in a maxillofacial and oral surgical unit in Johannesburg
}

SADJ May 2021, Vol. 76 No. 4 p194 - p200

K Sukha', M Mabongo ${ }^{2}$

\begin{abstract}
Introduction

Maxillofacial and oral surgical (MFOS) audits are able to provide data to both current and prospective patients regarding the quality of care an institution is capable of providing. The more frequently performed MFOS procedures can be determined and the allocation of funding and resources can therefore be achieved more appropriately.
\end{abstract}

\section{Aims and objectives}

To conduct an audit to evaluate the workload and scope of practice of the MFOS unit of the Charlotte Maxeke Johannesburg Academic Hospital (CMJAH) for the year 2015 by quantifying MFOS conditions and the respective treatment modalities.

\section{Design}

The study was retrospective and cross-sectional.

\section{Methods}

Data was retrieved from the patient logbook of the unit which was then entered into a Microsoft Excel Spreadsheet. Pie graphs and bar charts representing the data were then generated.

\section{Results}

A total of 1750 patients were treated in the unit. The male to female ratio was $1.3: 1$ and the majority of these patients were in their $3^{\text {rd }}$ and $4^{\text {th }}$ age decade. Most patients required a tooth extraction mainly for an impacted $3^{\text {rd }}$ molar.

\section{Conclusions}

Dentoalveolar surgery was the most commonly performed procedure followed by the treatment of facial fractures. Pathological and other MFOS conditions were

Author affiliations:

1. Kamal Sukha: BDS (Wits); MSc (Dent) (Wits), Dentist in Private Practice, South Africa.

2. Mzubanzi Mabongo: STD (Unitra); B Dent (Medunsa); BDS (Wits); MChD (MFOS)(UWC); Cert. Orofacial pain (Rutgers, USA); FCFS (Bangalore, India), Head of Department, Department of Maxillofacial and oral surgery, University of Pretoria, Pretoria, South Africa. ORCID Number: 0000-0001-9060-3996

Corresponding author: Kamal Sukha

Dentist in Private Practice, South Africa.

P.O. Box 709, Lenasia, 1820.

Email: dr.ksukha@gmail.com

Author contributions:

1. Kamal Sukha: Principal author - $50 \%$

2. Mzubanzi Mabongo: Second author - $50 \%$ less commonly encountered. The CMJAH MFOS unit treats a high volume of patients according to comparisons with global studies.

\section{INTRODUCTION}

Johannesburg is the capital city of the Gauteng Province, which is the economic hub of the Republic of South Africa. The Charlotte Maxeke Johannesburg Academic Hospital (CMJAH) based in Parktown is one of the teaching hospitals of the Faculty of Health Sciences of the University of the Witwatersrand, Johannesburg.

The hospital's Maxillo-Facial and Oral Surgical (MFOS) department/unit treats a wide spectrum of diseases, injuries and defects affecting the head, neck, jaw bones, face and the hard and soft tissues of the oral cavity. These procedures are performed under both local and general anaesthesia. The unit manages patients referred from healthcare facilities in Gauteng as well as surrounding provinces and also treats citizens of the Southern African Development Community (SADC) countries that lack the necessary skilled professionals, facilities and resources.

Clinical/surgical audits are widely used as a strategy for improving professional practice. Healthcare providers upon given feedback that their clinical practice is below standard are forced to modify that practice. A paper that reviewed a total of 140 studies concluded that audits lead to small but potentially important improvements in professional practice. ${ }^{1}$ A systematic review of surgical audits identified an important relationship between surgical audits and reducing the cost of healthcare by facilitating the provision of information and transparency on the performance of hospitals.

This knowledge allows for improvement in the quality of care, which then leads to fewer complications and ultimately less cost. ${ }^{2}$ Auditing provides information regarding the more frequently performed procedures within a surgical unit. A stricter control can thus be kept on the purchasing of instruments and consumable items as more funding can be allocated to the more commonly used products.

\section{LITERATURE REVIEW}

A number of audits conducted in various parts of the world have been reported in literature. A five-year audit of the MFOS department of Calabar Teaching Hospital 
in Nigeria revealed that a total number of 1,437 patients were treated from 2005 to 2009. ${ }^{3}$ Adebayo et al. reported results of another audit that was conducted in a Military hospital in Port Harcourt, which is part of the Niger Delta region of Nigeria. ${ }^{4}$ Both hospitals received referrals from a broad surrounding geographic area but the hospital in Port Harcourt supposedly only treated Nigerian military personal, explaining its lower patient volume. ${ }^{3,4}$ Adebayo et al. also found that the majority of patients treated at the Port Harcourt hospital could not afford specialised care, hence the low percentage of procedures that were actually performed. ${ }^{4}$

An audit in the Muhimbili National Hospital in Dar es Salaam found that 456 patients visited the MFOS unit for treatment over a period of six years. ${ }^{5}$ The authors considered this volume as significantly high even though the population of Tanzania is currently estimated at 51.04 million and the hospital was the only centre providing specialist MFOS services in the country.

Asian studies comparitively reveal a much higher number of individuals who required MFOS treatments. A study at the Dhaka Dental College showed a total number of 768 patients who were treated in the year $2012 .^{6}$ Researchers of the Kyber College of Dentistry in Peshawar, Pakistan similarly found that a high number of patients were seen in the hospital's MFOS unit. ${ }^{7}$ A total number of 2,764 patients were treated over a two-year period (January 2006 to December 2007) at an average of 1,382 patients per annum.

The studies cited above show that smaller volumes of patients are managed in African nations as compared to certain Asian countries. ${ }^{3-7}$ One can attribute the high volumes of patients in Asian nations due to their extremely high populations. The Nigerian authors Adebayo et al. suggest that their low patient volume was attributed to a lack of centres that are able to provide MFOS treatments. ${ }^{4}$ They also deduced that more patients actually had MFOS conditions during those five years but due to the lack of skilled professionals and appropriate screening, referral was not possible.

The study of most significance was a six-month comparison of the MFOS departmental statistics at the Chris Hani Baragwanath Hospital also in Johannesburg $(\mathrm{CHBH})$ for the years 1987 and 2007.8 Damtew et al. studied how MFOS practice had changed in South Africa over a period of 20 years in terms of both patient numbers and spectrum of conditions. A total number of 609 patients were treated in the MFOS unit during the first six months of 2007 . This number had increased from 445 in the year 1987 .

As far as conditions are concerned, Islam et al. of the Dhaka Dental College reported that maxillo-facial injury was most common followed by pathological and infective conditions. ${ }^{6}$ Uddin et al., whose audit was at the same institution found that the incidence of cleft lip and palate was considerably high. ${ }^{9}$ Even though both Nigerian studies described trauma-related conditions as the highest incidence in their facilities, there were also a considerable number of pathologic cases ranging from fibroosseous lesions, salivary gland tumours, cysts, both odontogenic and non-odontogenic tumours as well as septic conditions such as osteomyelitis.,

The British Association of Maxillo-Facial and Oral Surgeons First National Audit report in September 2010 presented a very broad scope of practice for specialists in the United Kingdom. ${ }^{10}$ The majority of participants practiced dentoalveolar surgery which included the removal of impacted wisdom teeth, trauma-related procedures e.g. open reduction with internal fixation (ORIF) of the mandible, and minor oral medicine procedures such as the treatment of lichen planus.

More than $80 \%$ of British surgeons were also involved in managing benign salivary gland tumours and cutaneous basal cell carcinomas. Surveys into changing trends in the scope of MFOS practice in Australia during 1990 and 2000 revealed that dentoalveolar surgery was the most commonly performed procedure in both eras. ${ }^{11,12}$ Brennan et al. observed that there was an increase in the rate of provision of services per specialist visit in the year 2000 as compared to 1990. ${ }^{11}$

There were significantly higher numbers of treatments of MFOS pathology, reconstructive surgery, implantology, bone grafting and orthognathic procedures by dual qualified surgeons (those who held both medical and dental degrees). ${ }^{10-12}$ The scope of practice in other parts of the world was much broader compared to Africa, as the treatment of congenital deformities and oral malignancies were more commonly practiced. ${ }^{3-12}$

\section{METHODS AND MATERIALS}

The research report is a retrospective, cross-sectional study of patients managed in the Maxillo-Facial and Oral Surgical (MFOS) unit of the Charlotte Maxeke Johannesburg Academic Hospital (CMJAH). The sample size consists of all patients (in-patients and out-patients) treated in the unit over a one-year period ( $1^{\text {st }}$ January 2015 to $31^{\text {st }}$ December 2015).

Data was retrieved from the statistics of the CMJAH MFOS department which included a theatre logbook for cases treated under general anaesthesia and a patient register for cases treated under local anaesthesia at the Wits Dental Hospital (located in the CMJAH). The data collected was comprised of patient age, gender, month of procedure, diagnosis of condition, anatomical site of condition (for trauma and pathology) and the treatment/procedure performed. The data was entered into a Microsoft Excel Spreadsheet where graphical representation was generated.

The diagnosis of MFOS conditions was categorised into the following:

1. Trauma: This includes fractures of the facial skeleton and lacerations of facial and oral soft tissues as a direct result of trauma.

2. Pathology: including all tumour and tumour-like conditions including cystic lesions of the jaw bones and oral soft tissues.

3. Teeth requiring extraction: This includes all impacted, carious and mobile teeth. Also includes teeth extracted prior to radiation therapy. NB: The patient 
number and not the number of teeth extracted was recorded.

4. Sepsis: including all abscesses, cellulitis and necrotic conditions.

5. Post-operative complications: This includes all dry/ septic sockets, oro-antral communications, displacement of teeth into ectopic areas and septic hardware after ORIFS (infected fractures).

6. Temporo-mandibular joint disorders (TMD) including ankylosis and bruxism resulting in trismus and myofascial symptoms.

7. Facial deformities and malocclusions.

8. Edentulous/partially edentulous patients requiring implants.

Treatments/procedures performed were categorised as follows:

1. Treatment of fractures of the facial skeleton either by open reduction with internal fixation (ORIF) or by closed reduction with inter-maxillary fixation (CRIMF).

2. Dentoalveolar surgery including the simple extraction of carious and mobile teeth as well as the surgical removal of impacted and unerupted teeth and full/ partial dental clearances prior to or during radiation therapy.

3. Oral soft tissue surgery.

4. Incisional and excisional biopsies.

5. Incision, drainage and debridement of sepsis.

6. Jaw reconstruction/bone grafts.

7. Orthognathic and orthodontic surgery.

8. Mandibular resections.

9. Temporo-mandibular joint (TMJ) surgery.

10. Implant placement.

11. Treatment of post-operative complications including the treatment of dry sockets, closure of oro-antral communications, removal of teeth displaced into the antra and removal of septic hardware and wires.

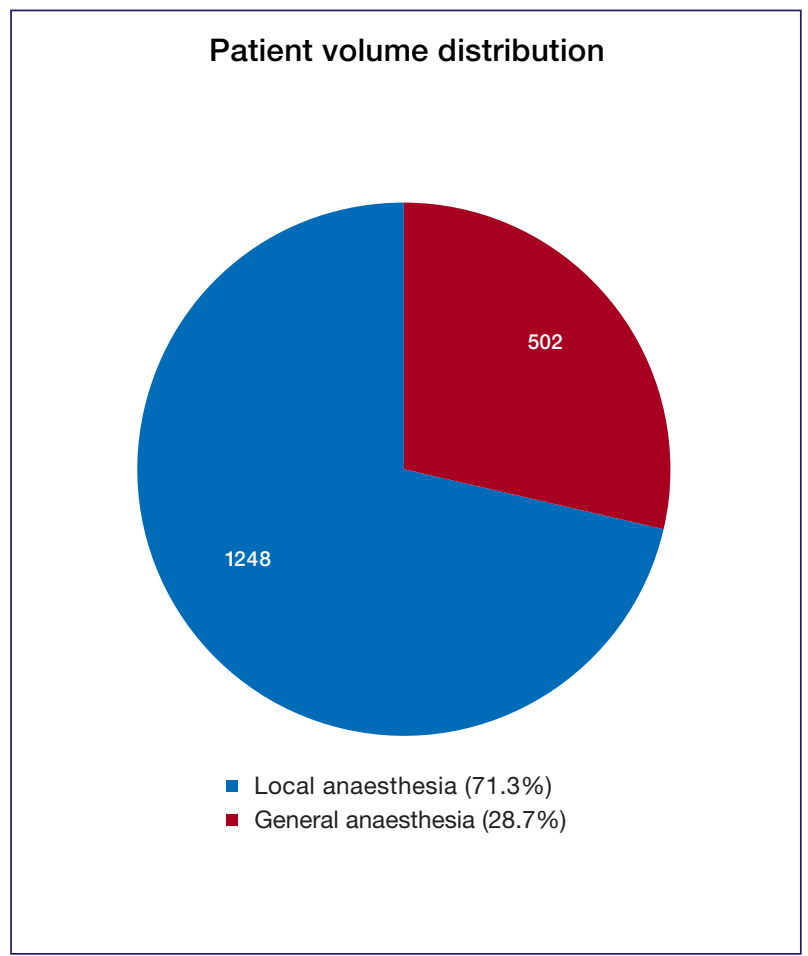

Figure 1. Pie chart demonstrating the volume distribution of patients treated in the unit based on the type of anaesthesia administered.

\section{Ethical considerations}

An application was made to the University of the Witwatersrand Committee for Research on Human Subjects (Medical) who approved the research protocol unconditionally (Clearance Certificate Number M160744). Permission for using the hospital and Wits Dental School's statistics was sought from the School of Oral Health Science Hospital Research Committee.

\section{RESULTS}

\section{Workload}

A total number of 1,750 patients were treated as elective cases in the CMJAH MFOS unit for the year 2015 ( $1^{\text {st }}$ January till $31^{\text {st }}$ December). Five hundred and two patients (502) were treated under general anaesthesia and 1,248 were treated as day cases under local anaesthesia. The distribution of patients with regard to treatment under general and local anaesthesia is presented in Figure 1.

\section{Gender}

A total number of 995 (56,8\%) males and 755 (43,2\%) females were treated in the unit during the year 2015 at a ratio of 1.3:1. For the 502 patients treated under general anaesthesia, 345 were males and 157 were females at a ratio of $2.2: 1$. The 1,248 patients treated under local anaesthesia, on the other hand had a relatively equal gender distribution at a ratio of 1.1:1.

\section{Age}

The age range of patients treated in the unit was from the $1^{\text {st }}$ to the $9^{\text {th }}$ decade with a mean age of 31,5 years (31,2 years for general anaesthesia and 31,8 years for local anaesthesia). Patients in their $3^{\text {rd }}$ and $4^{\text {th }}$ decades were more commonly seen under both general and local anaesthesia.

The two extremes of age (very young and very elderly) had a very low frequency. There were 18 patients from both categories whose age was unrecorded and could not be traced due to unrecorded file numbers in the patient register. Figure 2 graphically demonstrates the distribution of patients according to their age group and volumes.

\section{Age of distribution in decades}

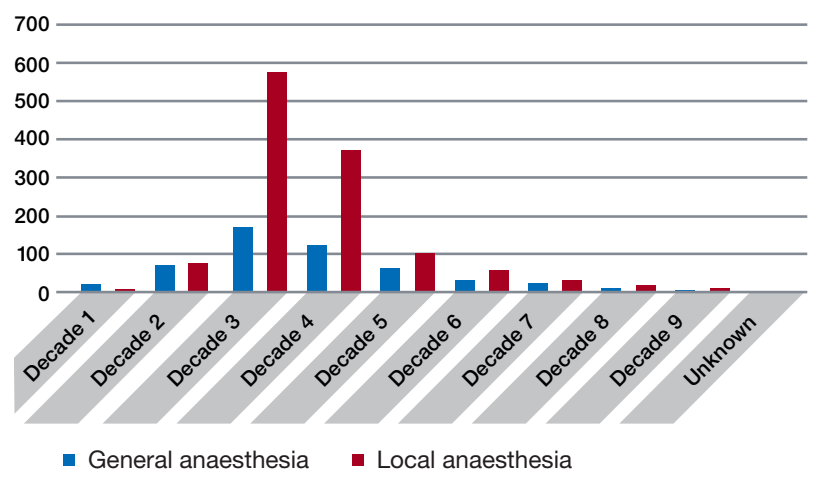

Figure 2. Bar graph demonstrating the age distribution in decades. 


\section{Conditions}

The percentage distribution of MFOS condtions are graphically represented in Figure 3. A certain percentage of patient conditions were not recorded in the patient register. This category is labelled as "unknown" and comprised $3,7 \%$ of all conditions. One patient developed general anaesthetic complications after the induction phase and could not be operated on. This category is labelled as "cancellations" in Figure 3 and comprised 0,4\% of all conditions.

\section{Pattern of trauma and tumour/ tumour-like conditions}

The percentage distribution of the specific anatomical sites that were affected is graphically demonstrated in Figures 4 and 5 below. Certain patient records (2,4\%) had unrecorded anatomical sites labelled as "unknown" on Figure 5. Sites for pathological conditions that were biopsied and treated under local anaesthesia are not included as they were not recorded in the patient register.

\section{Procedures/treatments}

The percentage distribution of all procedures performed (both under general and local anaesthesia) is graphically demonstrated in Figure 6 . Some procedures were not documented and represented $0,8 \%$ of the total.

Note that the quantity of procedures/treatments exceeds the total volume of patients as multiple procedures were performed on certain patients.

\section{DISCUSSION}

The volume of patients treated in the CMJAH MFOS unit during 2015 seems to be significantly high $(1,750$ patients). Although no direct comparison can be made, as an audit of this nature has never previously been undertaken in the unit, anecdotal evidence suggests that the volumes have always been high which are comparable globally to audits conducted in Asian nations. ${ }^{5-7}$

Damtew et al. reported an increase in the patient volume at the $\mathrm{CHBH}$ due to the significant growth of the population within Johannesburg and the influx of foreign nationals from surrounding African countries. ${ }^{8}$ These factors could apply to the CMJAH as both hospitals are about $20 \mathrm{~km}$ apart. A study also demonstrated how there is an average delay of 20 days at the CMJAH between an injury (facial fracture) and its treatment. ${ }^{19}$ This is further evidence of the high patient volumes.

The majority of these patients were treated under local anaesthesia due to the intense patient volume. It is simply not possible for all these cases to be treated under general anaesthesia. Patient comfort and satisfaction for dentoalveolar surgery, sepsis drainage as well as incisional and excisional biopsies can be attained through effective local anaesthesia. The treatment under local anaesthesia reduces the pressure placed on the elective theatre slate and allows for substantially more patients to be treated. It also allows preference for the more severe cases to be treated under general anaesthesia.
The majority of patients treated in the unit were in their $3^{\text {rd }}$ and $4^{\text {th }}$ decade with a mean age of 31,5 years. This result concurs with a study that found how the majority of individuals require $3^{\text {rd }}$ molar surgery after the age of $20 .{ }^{14}$ Maxillofacial trauma was shown to occur in individuals of the same age group in western societies and the author attributed alcohol abuse predominantly in this group as a pivotal factor in the increased number of road accidents and incidents of interpersonal violence globally. ${ }^{15}$

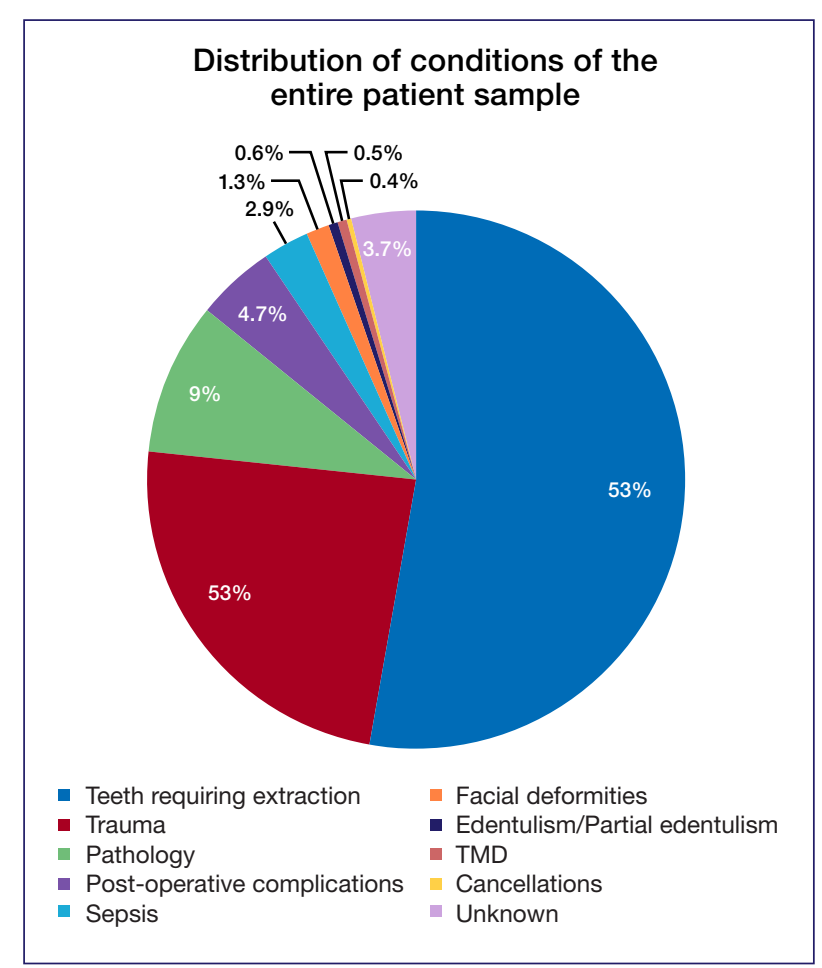

Figure 3. Pie chart demonstrating the distribution of conditions of all patients seen in the unit.

\section{Anatomical site distribution/pattern of trauma} for cases treated under general anaesthesia

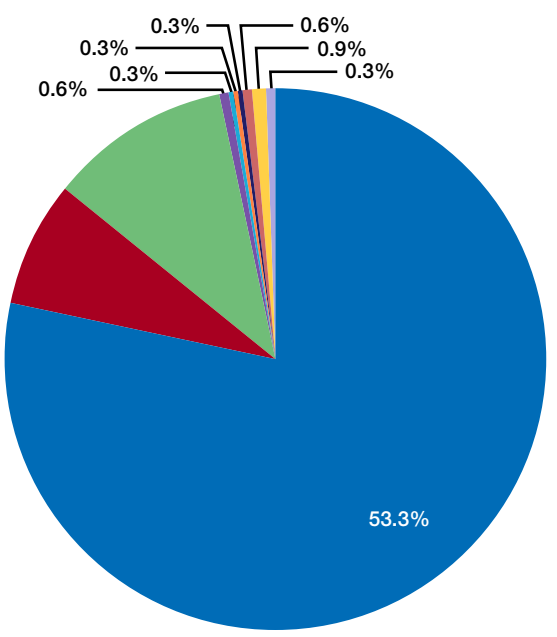

$$
\begin{array}{ll}
\text { - Mandibular fractures } & \text { - Supra orbital fractures } \\
\text { - Maxillary fractures } & \text { - Infraorbital fractures } \\
\text { - Zygomatic complex } & \text { - Orbital floor fractures } \\
\text { fractures } & \text { Facial tissue lacerations } \\
\text { - Pan facial fractures } & \text { Tongue lacerations } \\
\text { - Frontal bone fractures } &
\end{array}
$$

Figure 4. Pie chart demonstrating the anatomical site distribution/pattern of trauma for cases treated under general anaesthesia. 
In South Africa, mandibular fractures occurred mainly in patients between the age of 20 and 40 at the CMJAH according to Desai et al. ${ }^{16}$ The paper also noted that $86 \%$ of these patients who were treated in this unit during a six-month period in 2004 were victims of interpersonal violence.

The male to female ratio for the entire sample was 1.3:1. The majority of global trends suggest that males are more commonly affected by maxillo-facial conditions..$^{3-8}$ Lee et al. described trends where males more commonly sustained facial fractures due to higher involvement in interpersonal violence and motor vehicle accidents. ${ }^{15}$

The majority of procedures performed under local anaesthesia included dentoalveolar surgery primarily for surgical removal of the $3^{\text {rd }}$ molar. There is however no conclusive evidence that identifies whether males or females more commonly undergo $3^{\text {rd }}$ molar surgery. According to a study conducted in Pakistan by Khan et al., males were more likely to undergo surgical removal of impacted wisdom teeth. These authors do however state that studies conducted in Malaysia and Saudi Arabia showed contrary results. ${ }^{14}$ These conflicting findings can be attributed to various factors such as the difference in geographic areas and the diverse ethnicity of the area's citizens.

Fifty three percent (53\%) of the entire patient sample required tooth extraction/s, which predominantly included $3^{\text {rd }}$ molar surgery. Traumatic facial injuries were also very prevalent. Audits conducted in Africa demonstrate how MFOS surgeons frequently treated facial fractures. $3,4,5,8,9,17,18$

This is contrary to Asian studies where specialists are more involved in treating pathological conditions. , $^{7,9}$ The high volumes of trauma seen in the CMJAH unit is due to South Africa's high incidence of violent crime and road accidents.

Pathological conditions occurred in $9 \%$ of the patients in this audit. Specific tumour types were not included as part of the study. Anecdotal evidence suggests that a high prevalence of ameloblastomas affecting the mandible occur frequently in the unit. This supports our finding that the majority of pathological lesions occured in the mandible. The management of malignant conditions such as squamous cell carcinomas were commonly performed in Bangladesh. 6,9

A British study also reported that head and neck oncologic conditions were treated by maxillofacial and oral surgeons. ${ }^{10}$ According to the CMJAH policy however, malignancies are not directly treated in the unit. A full dental clearance if indicated is requested by either the oncology or ENT surgery departments, which subsequently provide direct management in terms of surgery, radiation or chemotherapeutic treatments.

Post-operative complications and sepsis were relatively low. Damtew et al. also reported a slight decrease in sepsis from $8 \%$ in 1997 to $6 \%$ in 2007 at the $\mathrm{CHBH}^{8}$ A possible explanation for this observation is that most patients are now self-aware and educated with regard to their health and seek treatment before conditions progress to severe sepsis. We also assume that the incidence of HIV-related deaths has also decreased in South Africa from 2005 due to the rollout of anti-retroviral medication. ${ }^{19}$ This view is supported by an American study which found that the epidemiology of sepsis in patients with HIV has changed significantly with advancements in HAART (highly active antiretroviral therapy).

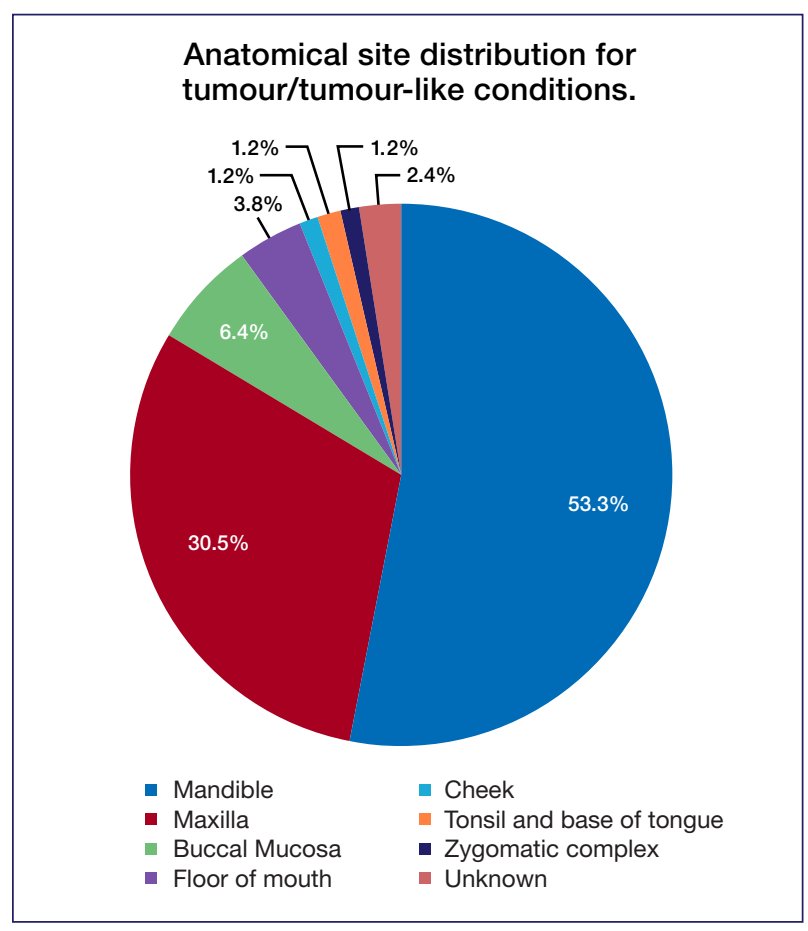

Figure 5. Pie chart demonstrating the anatomical site distribution for tumour/tumour-like conditions.

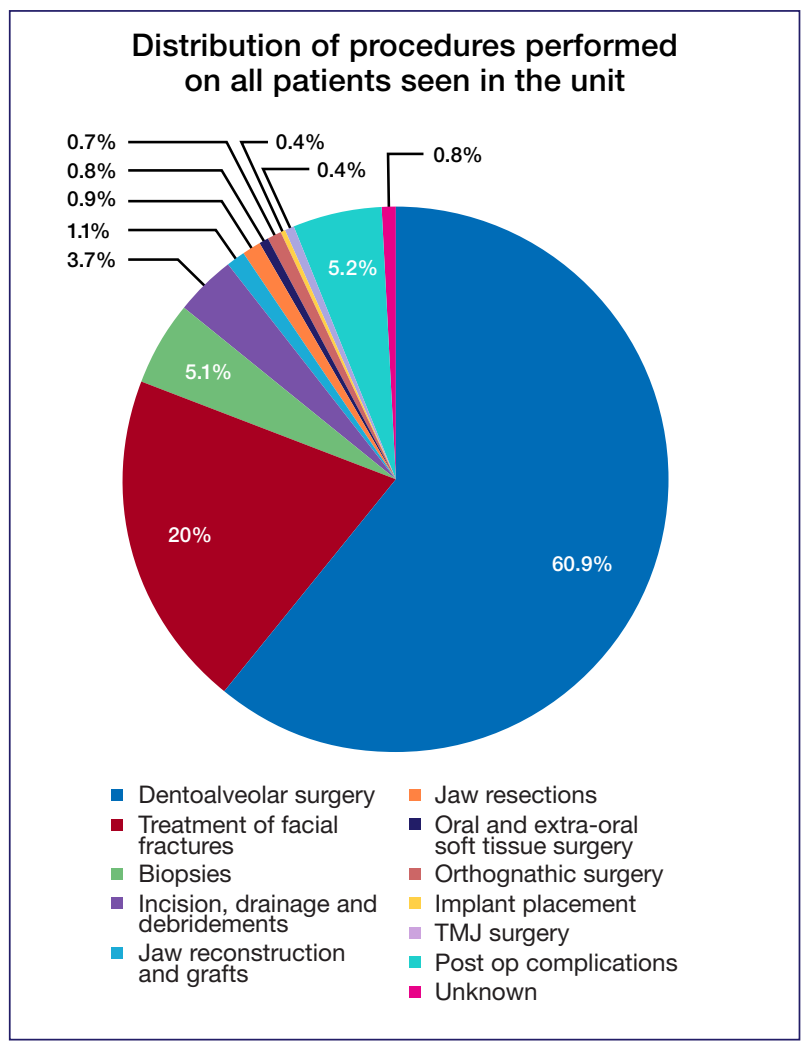

Figure 6. Pie chart demonstrating the distribution of procedures performed on all patients seen in the unit. 
These authors found a decrease in ICU admissions of HIV patients with septic conditions and an increase in survival rates. ${ }^{20}$

Isolated conditions such as facial deformities, edentulism and TMD were not commonly encountered by the unit and only treated under general anaesthesia. We suspect that these conditions were seldomly encountered due to exorbitant costs associated with their treatment, particularly for implant placements.

Dentoalveolar surgery, which included 1,162 procedures, was the most commonly performed treatment type on the entire patient sample. This elevated procedure type can be credited to the increased number of patients who required $3^{\text {rd }}$ molar surgery and other complicated tooth extractions. Developed nations like Australia also confirm a high prevalence of dentoalveolar surgery in their institutions. ${ }^{11,12}$

Brennan et al. reported dentoalveolar surgery rates of as high as $60-70 \%$ of all procedures performed by MFOS surgeons in Australia, followed by trauma surgery which ranked a very distant second. ${ }^{11}$ ORIFS performed under general anaesthesia constituted 55\% of all facial fractures treated. ORIFS are normally indicated in patients with severe injuries and severe displacement to restore previous anatomic relationships. Edentulous and partially edentulous patients also benefit from ORIFS as they have a lack of stable occlusal contacts for closed reductions. ${ }^{21}$

The indications of CRIMF include moderate displacement of the fractured fragments and a presence of stable occlusal contacts. The pattern of trauma in the unit revealed that the mandible was the most commonly fractured bone. Lee of the University of Christchurch found that the mandible was commonly fractured after traumatic incidents especially in cases of interpersonal violence, which could explain our finding. ${ }^{15}$

The zygomatic bone was fractured in $10,8 \%$ of patients followed by the maxilla at $7,5 \%$. This pattern explains why a significant number of CRFMS for mandibular fractures and certain maxillary fractures could be carried out. Isolated fractures of the orbital floor and orbital rim were treated by ORIF.

Biopsy of tumour/tumour-like conditions was performed on $5,1 \%$ of individuals. There is an atypical pattern where $9 \%$ of the total sample of patients presented with a pathological lesion and only $5,1 \%$ of these lesions were biopsied. We again speculate that a certain number of patients were referred to specialists in other medical disciplines for definitive management of their conditions and biopsies were not performed on them.

The frequency of orthognathic surgery and implant placements were low. Global studies from developing nations suggest that implant placements were not commonly performed due to the high costs involved. ${ }^{3-9}$ Bezerra et al. in a Brazilian hospital also reported a low incidence of dental implant placements between the years 2000 and 2006. It was also noted that patients within the private healthcare system more commonly received den- tal implants due to them being able to afford the exorbitant costs associated with the procedure. ${ }^{22}$

Similarly, implant placements at the CMJAH were not frequently done due to financial implications associated with components purchased from the manufacturer. Jaw resection normally performed to remove tumours such as ameloblastomas, fibro-osseous and cystic lesions and the subsequent re-constructive surgery were infrequently performed due to a lack of patients presenting with these conditions.

\section{CONCLUSION}

According to global workloads and trends, the CMJAH MFOS unit treats a significantly high number of patients. The scope of MFOS practice in the unit is relatively broad but the frequency of advanced procedures can be considered as insufficient. Future studies should consider data that includes the private sector to establish a broader national trend.

\section{Recommendations}

1. The Department of Health needs to consider activation and budgeting for the previous MFOS units in other government hospitals (Thembisa, Leratong, Natalspruit and Helen Joseph) to reduce the workload of the current operating units.

2. The Department of Health also needs to consider revising its current budget to the hospital. An increase in funds would improve resources and allow for more advanced MFOS to be practiced.

3. The workload of the unit could be reduced by training more dental practitioners in primary healthcare procedures such as the treatment of mandibular fractures and dentoalveolar surgery. This would free up time for registrars to broaden their scope of MFOS practice and also decrease the waiting time for patients.

4. For the adequate training of registrars, a certain amount of time should be dedicated to consultants in private practices who are more commonly perform advanced MFOS procedures.

5. A trauma fellowship could be included in the current teaching curriculum due to the high prevalence of trauma-related injuries and procedures.

6. Record-keeping within the unit needs to be improved possibly by computerisation to decrease the incidence of incomplete records and allow simplicity for future surgical audits.

\section{References}

1. Ivers N, Jamvedt G, Flottorp S, et al. Audit and feedback: effects on professional practice and healthcare outcomes. The Cochrane database of systematic reviews. 2012; 13: CD000259.

2. Govaert JA, van Bommel ACM, van Dijk WA, et al. Reducing healthcare costs facilitated by surgical auditing: A systematic review. World Journal of Surgery. 2015; 39: 1672-80.

3. Anyanechi CE, Saheeb BD. Audit of the demographic patterns of oral and maxillofacial surgical cases in a Nigerian teaching hospital. International Journal of Medicine and Health Development. 2011; 16: 18-27. 
4. Adebayo ET, Ajike SO, Abite MG. Audit of oral and maxillofacial conditions seen at Port Harcourt, Nigeria. Annals of African Medicine. 2008; 7: 29-34.

5. Moshy J, Hamza O, Moshiro C. An audit of 6 years of oral and maxillofacial surgical conditions admitted for interventional treatment at Muhimbili National Hospital, Dar es Salaam - Tanzania. East and Central African Journal of Surgery. 2012; 17: $95-101$

6. Islam MA, Haider IA, Uzzaman MH, Tymur FR, Ali MS. One year audit of in-patient department of oral and maxillo-facial surgery Dhaka Dental College Hospital. J Maxillofac Oral Surg. 2016; 15: 229-35.

7. Rehman B, Din QU. Two years audit of maxillofacial surgery department at Khyber College of Dentistry, Peshwar. Pak Oral Dent J. 2009; 29: 13-8.

8. Damtew MM. Changing trends in maxillo-facial and oral surgery at Chris Hani Baragwanath Hospital: A comparison between two time periods in 1987 and 2007. <https://wired space.wits.ac.za> [Accessed 2 February 2017].

9. Uddin N, Ahmed MU, Haider IA, Moral AKMS. Annual clinical audit of Indoor, Dhaka Dental College and Hospital (January 2004 to December 2005). Bangladesh Journal of Dental Research and Education. 2015; 5: 37-9.

10. Rogers SN, Lowe D. British Association of Oral and Maxillofacial Surgeons first national audit in support of revalidation. Br J Oral Maxillofac Surg. 2011; 49: 478-9.

11. Brennan DS, Spencer AJ, Singh KA, Teusner DN, Goss AN. Practice patterns of oral and maxillofacial surgeons in Australia: 1990 and 2000. Int J Oral Maxillofac Surg. 2004; 33: 598-605.
12. Ricciardo P, Bobinskas A, Vujcich N, Nastri A, Goss A. Survey of Australasian oral and maxillofacial surgeons 2011 - scope and workforce issues. Int J Oral Maxillofac Surg. 2015; 44: 1569-73.

13. Porter M, Lownie M, Cleaton-Jones P. Maxillofacial injury: a retrospective analysis of time lapse between injury and treatment in a South African academic maxillofacial and oral surgery unit. S Afr J Surg. 2013; 51: 138-42.

14. Khan M, Mehbhoob B, Shajahan K. Extraction of wisdom teeth under general anaesthesia - A study. Journal of Kyber College of Dentistry. 2014; 5: 20-4.

15. Lee K. Global trends in maxillofacial fractures. Craniomaxillofac Trauma Reconstr. 2012; 5: 213-22.

16. Desai J, Lownie JF, Cleaton-Jones P. Prospective audit of mandibular fractures at the Charlotte Maxeke Johannesburg Academic Hospital. S Afr J Surg. 2010; 48: 122-6.

17. Oginni FO, Oladejo T, Alake DP, Oguntoba JO, Adebayo OF. Facial bone fractures in Ile-Ife, Nigeria: An update on pattern of presentation and care. J Maxillofac Oral Surg. 2016; 15: 184-90.

18. Oginni FO, Ugboko VI, Ogundipe O, Adegbehingbe BO. Motorcycle-related maxillofacial injuries among Nigerian intracity road users. J Oral Maxillofac Surg. 2006; 64: 56-62.

19. Statistics South Africa. < http://www.statssa.gov.za> [Accessed 2 February 2017].

20. Mayr F, Yende S, Angus D. Epidemiology of sepsis. Virulence 2014; 5: 4-11.

21. Chircanovic BR. Open vs. closed reduction: Comminuted mandibular fractures. Oral Maxillofac Surg. 2013; 17: 95-104.

22. Bezerra MF, Avelar RL, de Oliveira RB, Studart-Soares EC, Pretto MS. Assessment of the oral and maxillofacial surgery services in a teaching hospital. The Journal of Craniofacial Surgery. 2011; 22: 50-3

\section{NOTICE of the $21^{\text {st }}$ ANNUAL GENERAL MEETING (AGM)}

Notice is hereby given that the $21^{\text {st }}$ Annual General Meeting of Members (AGM) of The South African Dental Association (SADA) NPC, will be held on Thursday, 10 June 2021 at $18 \mathrm{~h} 00$, which will be conducted virtually and electronically on this date through the Zoom virtual meeting platform or similar digital platform. The Agenda with any supporting documents for the meeting will be posted on the SADA website.

SADA is your Association and your voice counts.

KC Makhubele

Chief Executive Officer

April 2021

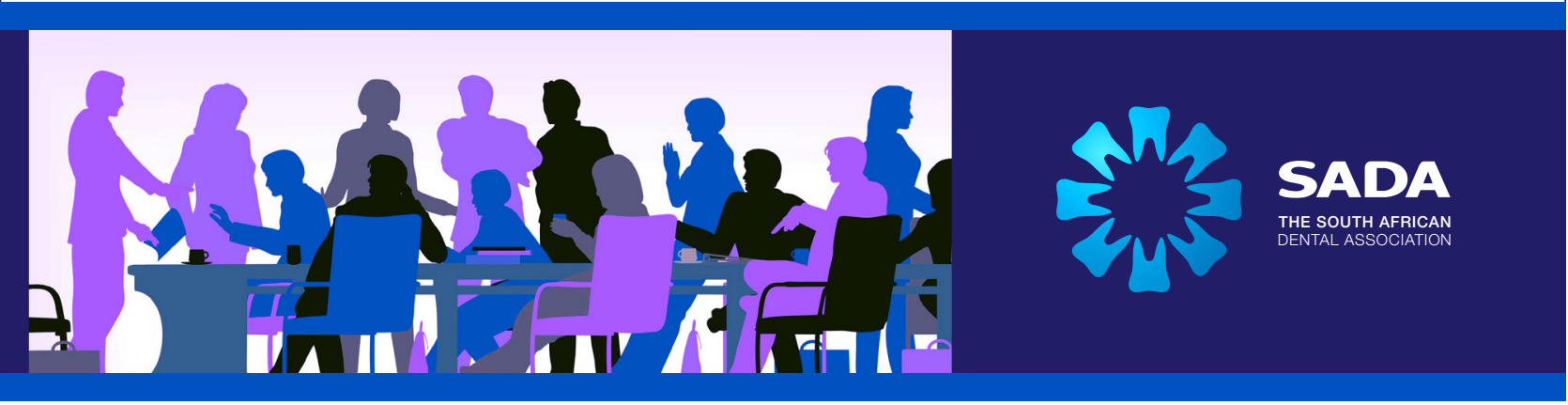

